\title{
In-vitro Characterization of an L-Kynurenine-Responsive Transcription Regulator of the Oxidative Tryptophan Degradation Pathway in Burkholderia xenovorans
}

\author{
Richard S Hall ${ }^{1}$, Ricardo Martí-Arbona ${ }^{1}$, Scott P Hennelly ${ }^{1}$, Tuhin S Maity ${ }^{1}$, Fangping Mu ${ }^{1}$, John M Dunbar ${ }^{1}$, \\ Clifford J Unkefer ${ }^{1} \&$ Pat J Unkefer ${ }^{1}$ \\ ${ }^{1}$ Los Alamos National Laboratory, Los Alamos, NM 87545, United States \\ Correspondence: Pat J Unkefer, Los Alamos National Laboratory, Los Alamos, NM 87545, United States. \\ E-mail: punkefer@lanl.gov
}

Received: May 3, 2012 Accepted: June 18, 2013 Online Published: July 2, 2013

doi:10.5539/jmbr.v3n1p55 URL: http://dx.doi.org/10.5539/jmbr.v3n1p55

\begin{abstract}
To study the transcriptional regulation of oxidative tryptophan degradation in Burkholderia, we used comparative genomics that focused on the operon containing the genes annotated as $k y n A, k y n U$ and $k y n B$. In all sequenced $\beta$-proteobacteria to-date, including Burkholderia, Ralstonia, Collimonas, and Cupriavidus species, there is a conserved AsnC/Lrp family transcriptional regulator (TR) gene located upstream and in the opposite strand of the operon encoding for the oxidative tryptophan degradation genes. In Burkholderia xenovorans the TR is Bxe_A0736. GST-Bxe_A0736 binds L-kynurenine with greater affinity and specificity than any other amino acid or tryptophan degradation product with a dissociation constant of $\sim 82 \pm 11 \mu \mathrm{M}$. DNaseI footprinting suggested that Bxe_A0736 protects a set of four degenerate, palindromic sequences within the intergenic region between Bxe_A0735 (kynB) and Bxe_A0736. The optimal consensus sequence obtained by analysis for these sites, ATATTCCGAATAT, closely resembles the sequence obtained with a protein binding microarray. Under our fluorescence anisotropy experimental conditions, $1 \mathrm{mM} \mathrm{L}$-kynurenine increased the affinity of Bxe A0736 for a portion of its promoter region. Our results are consistent with Bxe_A0736 acting as the TR that promotes the transcription of the oxidative tryptophan degradation genes in the presence of L-kynurenine while inhibiting the transcription of its own gene.
\end{abstract}

Keywords: Bxe_A0736, L-tryptophan degradation, kynurenine, fluorescence anisotropy, Burkholderia xenovorans, TR, transcriptional regulator, FAC-MS, frontal affinity chromatography coupled to mass spectrometry, COG, clusters of orthologous groups, PBM, protein binding microarray, footprinting, TRE, transcriptional regulator effector

\section{Introduction}

The kynurenine pathway oxidatively degrades L-tryptophan to provide important precursors for molecules such as quinolobactin siderophores and quorum signaling quinolones. $\mathrm{KynA}, \mathrm{KynB}$ and $\mathrm{KynU}$ catalyze the first steps in the kynurenine pathway. The products of these first pathway steps are formic acid, anthranilic acid and L-alanine, where N-formyl-L-kynurenine and L-kynurenine are intermediates (Figure 1). L-Tryptophan oxidation activity is induced by L-kynurenine in the $\gamma$-proteobacterium Pseudomonas fluorescens (Palleroni \& Stanier, 1964). Mutants of the L-kynurenine pathway in P. aeruginosa had a reduced ability to kill Staphylococcus aureus, possibly through a reduced production of 4-quinolones such as the Pseudomonas quinolone signal (PQS) 2-heptyl-3-hydroxy-4-quinolone (Farrow \& Pesci, 2007). The L-kynurenine route of tryptophan degradation has been implicated as a contributor to bacterial pathogenesis through quinolobactin siderophore biosynthesis (Matthijs et al., 2004) and L-kynurenine has been implicated in contributing to a state of infectious tolerance within the host (Belladonna, Orabona, Grohmann, \& Puccetti, 2009).

The $\beta$-proteobacterium Burkholderia xenovorans shares much in common with pathogens such as B. mallei, $B$. pseudomallei, $P$. aeruginosa and $P$. fluorescens. These similarities are evident in the genomic organization around the genes kynA, kynB and kynU (Figure 2). In B. xenovorans, the TR Bxe_A0736 is located 130 bases upstream of the start codon for $k y n B$ on the opposite strand. Bxe_A0735 is a very well conserved TR annotated as asnC. Proteins sharing a greater than $60 \%$ sequence identity, with similar operon organization, are present 
throughout $\beta$-proteobacteria, including Burkholderia species, Cupriavidus taiwanensis, Cupriavidus metallidurans, Ralstonia solanacearum, Ralstonia pickettii, Ralstonia metallidurans, Ralstonia eutropha, and in the $\gamma$-proteobacteria Pseudomonas fluorescens and Pseudomonas aeruginosa. The AsnC/Lrp family TR PA2082 from $P$. aeruginosa is responsible for the L-kynurenine-dependent transcription of the kynurenine pathway genes kynA and kynB and should be annotated as kynR (Knoten, Hudson, Coleman, Farrow \& Pesci, 2011). KynR from $P$. aeruginosa and Bxe_A0736 share a $66 \%$ protein sequence identity.<smiles>NC(Cc1c[nH]c2ccccc12)C(=O)O</smiles>

L-tryptophan

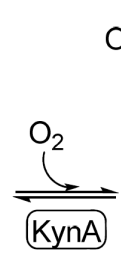

$\mathrm{N}$-formyl L-kynurenine<smiles>Nc1ccccc1C(=O)C(N)C(=O)O</smiles>

L-kynurenine

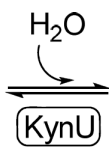

$\mathrm{HO}$<smiles>CC(N)C(=O)O</smiles>

anthranilic acid L-alanine

Figure 1. The kynurenine pathway of oxidative degradation of L-tryptophan. The products of the pathway are anthranillic acid, alanine and formic acid

The AsnC/Lrp family of TRs is named after two of the three TRs in this family that are present in E. coli. AsnC binds L-asparagine (de Wind, de Jong, Meijer, \& Stuitje, 1985; Kolling \& Lother, 1985) to control the transcriptional repression of asparagine synthetase while acting as an autorepressor of its own transcription (Kolling \& Lother, 1985). Lrp binds L-leucine, controlling a global metabolic response, particularly in amino acid synthesis and degradation in response to feast or famine (Brinkman, Ettema, de Vos, \& van der Oost, 2003; Calvo \& Matthews, 1994; Landgraf, Wu, \& Calvo, 1996). The third family member, YbaO has no known effector. Effectors have been indentified for other members of the AsnC/Lrp family including L-leucine for Lrp (Willins, Ryan, Platko \& Calvo, 1991), L-proline for PutR (Keuntje, Masepohl, \& Klipp, 1995), L-glutamate for Grp (Peekhaus, Tolner, Poolman, \& Kramer, 1995), L-methionine for MdeR (Inoue et al., 1997) and branched chain amino acids for BkdR (Madhusudhan, Lorenz, \& Sokatch, 1993).

X-ray crystal structures have shown that members of the AsnC/Lrp family of TRs form an octameric disc shape where the helix-turn-helix DNA binding domains are displayed on the outside of the structure (Koike, Ishijima, Clowney \& Suzuki, 2004; Ren et al., 2007; Thaw et al., 2006). Based on those structures, it has been proposed that DNA wraps around the perimeter of the octameric protein complex. This structure may explain why several of the TRs in this family have been shown to have rather long regions of DNA interacting with these TRs (Enoru-Eta, Gigot, Thia-Toong, Glansdorff, \& Charlier, 2000; Wang \& Calvo, 1993; Yokoyama, Ebihara, Kikuchi, \& Suzuki, 2005). The closest homologue of Bxe_A0736 with a crystal structure deposited to the Protein Data Bank is Lrp from Neisseria meningitides (Ren et al., 2007). X-ray crystal structures of Lrp showed an absence of significant conformational differences between L-leucine- bound Lrp and ligand-free Lrp. It was stated that effector binding appears to stabilize the octameric assembly of the TR (Ren et al., 2007). The mechanism of effector-controlled transcriptional regulation by members of the AsnC/Lrp family is in need of further study.

Reliable functional knowledge is lacking for many proteins. This is especially true for transcriptional regulators due to the difficulty in uncovering their function and mechanism. To characterize the TR Bxe_A0736 in $B$. xenovorans we utilized a battery of techniques for transcriptional regulator prediction and characterization, effector prediction, screening and characterization as well as DNA regulatory site identification and characterization. Here we report that TR Bxe_A0736 binds L-kynurenine but not other metabolites in tryptophan degradation and that it interacts with a rather long region of DNA that contains repeated pallindromic sequences that are found upstream of Bxe_A0735. We provide evidence in support of Bxe_A0736 as involved in regulating the oxidative tryptophan degradation genes kynA, $k y n B$ and $k y n U$.

\section{Methods}

\subsection{Sequence Similarity Network Analysis}

A sequence similarity network was constructed according to the method of Atkinson and coworkers (Atkinson, Morris, Ferrin, \& Babbitt, 2009). Their method of independent pairwise alignments between sequences allows functional relationships to be observed over very large sets of evolutionarily related proteins, such as members of 
specific COGs (clusters of orthologous groups). The COG database accessible on the NCBI website contains most of the predicted proteins in 66 genomes of unicellular organisms (Tatusov et al., 2003). According to the classification in this database, COG1522 contains the diverse members of the AsnC/Lrp TR family. The method requires that increasingly stringent BLAST E value cutoffs be tested until the desired degree of clustering is observed. At a BLAST $E$ value cutoff of $10^{-40}$, some clustering is achieved; the proline-binding PutR and the leucine-binding Lrp proteins grouped together and the glutamate-binding Grp from Zymomonas mobilis grouped with $\mathrm{YbaO}$ from $E$. coli, a TR of unknown function. At a BLAST $E$ value cutoff of $10^{-45}$, the different characterized functions for COG1522 separate into the distinct groups. This E value was used. The results are visualized using the organic layout in Cytoscape (Cline et al., 2007).

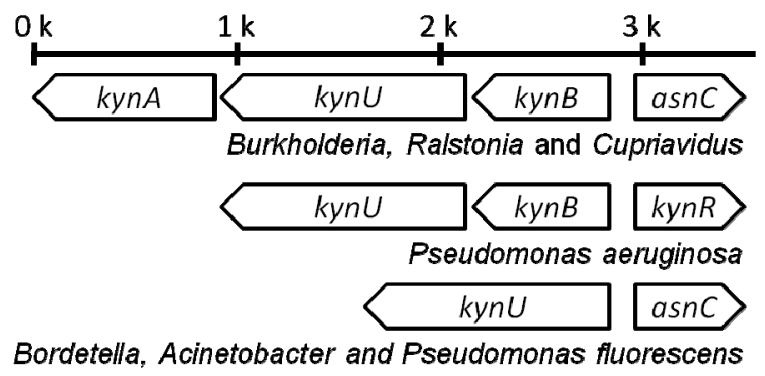

Figure 2. Genomic organization around kynA, kynB and kynU from several bacteria. The Burkholderia xenovorans TR Bxe_A0735 is annotated as asnC. The positions of TR-encoding genes annotated as $a s n C$ and kynR relative to other genes in the kynurenine pathway are conserved in these organisms

\subsection{Cloning and Protein Production}

\subsubsection{Cloning and Overexpression of GST-Bxe_A0736}

Bxe_A0736 was synthesized and sub-cloned into pET42a $(+)$ by Genscript with codon usage optimized for protein expression in $E$. coli (the optimized DNA sequence for the protein can be found in the supplemental material, Figure S1). The $P s h A \mathrm{I}$ and EcoRI cloning sites were utilized to incorporate an N-terminal GST protein fusion tag which is cleavable by Factor Xa digestion. The plasmid DNA was then transformed into ArcticExpress (DE3) competent cells from Agilent Technologies and plated onto an LB/Agar plate containing50 $\mu \mathrm{g} / \mathrm{mL}$ kanamycin. A single colony was used to inoculate LB media supplemented with $50 \mu \mathrm{g} / \mathrm{mL}$ kanamycin and $20 \mu \mathrm{g} / \mathrm{mL}$ gentamycin for overnight growth with shaking at $37^{\circ} \mathrm{C} .10 \mathrm{~mL}$ of the overnight culture was used to inoculate each liter of sterile LB broth in a 2 liter shaker flask and supplemented with $50 \mu \mathrm{g} / \mathrm{mL}$ kanamycin. The cells were incubated at $37^{\circ} \mathrm{C}$ with shaking until they yielded an O.D. of 0.5 at $600 \mathrm{~nm}$. The temperature of the growth chamber was then reduced to $15^{\circ} \mathrm{C}$ and isopropyl- $\beta$-d-thiogalactopyranoside (IPTG) was added to a final concentration of $0.5 \mathrm{mM}$.

\subsubsection{Cloning and Overexpression of Bxe_A0736-His 8}

The Bxe_A0736 gene was amplified by conventional PCR methods (manufacturer's protocol for Platinum Pfx DNA polymerase- Invitrogen) from the pET42a $(+)$ construct synthetized by Genscript (section 2.1.1). The Bxe A0736 gene was amplified using the primers: forward 5'-CATATGAATGCGATCTCCCTGGAC-3' and reverse 5'-CTCGAGGCGATCATTATGGGCAGC-3', containing NdeI and XhoI sites, respectively. The resulting PCR product was digested with the respective endonucleases and ligated into the pET42a $(+)$ vector at the NdeI and XhoI sites. The C-terminal His 8 tagged Bxe_A0736 (Bxe_A0736-His 8 ) encoding recombinant plasmid was transformed into ArcticExpress (DE3) competent cells from Agilent Technologies and plated onto an LB/Agar plate containing $50 \mu \mathrm{g} / \mathrm{mL}$ kanamycin. A single colony was used to inoculate LB media supplemented with $50 \mu \mathrm{g} / \mathrm{mL}$ kanamycin and $20 \mu \mathrm{g} / \mathrm{mL}$ gentamycin for overnight growth with shaking at $37^{\circ} \mathrm{C} .10 \mathrm{~mL}$ of the overnight culture was used to inoculate each liter of sterile LB broth in a 2 liter shaker flask and supplemented with $50 \mu \mathrm{g} / \mathrm{mL}$ kanamycin. The cells were incubated at $37^{\circ} \mathrm{C}$ with shaking until they yielded an O.D. of 0.5 at $600 \mathrm{~nm}$. The temperature of the growth chamber was then reduced to $15^{\circ} \mathrm{C}$ and isopropyl- $\beta$-d-thiogalactopyranoside (IPTG) was added to a final concentration of $0.5 \mathrm{mM}$. 


\subsubsection{Protein Purification}

After incubation at $15^{\circ} \mathrm{C}$ for 24 hours, the cells (expressing either GST-Bxe_A0736 or Bxe_A0736-His 8 ) were harvested with centrifugation at $3,000 \times g$ for $25 \mathrm{~min}$. The cells were then re-suspended in $10 \mathrm{mM} \mathrm{Na}_{2} \mathrm{HPO}_{4}, 1.8$ $\mathrm{mM} \mathrm{KH} \mathrm{PO}_{4}, 140 \mathrm{mM} \mathrm{NaCl}, 2.7 \mathrm{mM} \mathrm{KCl}, 1 \mathrm{mM}$ DTT, $1 \mathrm{mM}$ EDTA, pH 7.3 (Buffer A) supplemented with $100 \mu \mathrm{g} / \mathrm{mL}$ of the protease inhibitor phenylmethanesulfonyl fluoride and $1 \mathrm{unit} / \mathrm{mL}$ of DNase I. The cells were then lysed with sonication while being stirred on ice and then the cell debris was removed from solution by centrifugation at $14,000 \times g$ for $30 \mathrm{~min}$. The remaining solution was passed through a 0.45 um syringe filter and loaded onto an AKTA Purifier HPLC from GE Healthcare with two stacked $5 \mathrm{~mL}$ GSTrap FF HiTrap affinity columns for the GST-Bxe A0736 or two stacked $5 \mathrm{~mL}$ HisTrap HP (Ni) affinity columns for the Bxe_A0736-His 8 . The bound protein was washed with 10 column volumes of binding buffer and then eluted with a linear gradient $(0-50 \%)$ of $20 \mathrm{mM}$ Tris at $\mathrm{pH} 8.0$ containing $1 \mathrm{mM}$ DTT and $700 \mathrm{mM}$ glutathione for GST-Bxe_A0736 or with a 0-55\% gradient of Buffer A containing $700 \mathrm{mM}$ imidazole for Bxe_A0736-His s. In each separate case, the protein containing fractions were pooled and concentrated to about $10 \mathrm{~mL}$ and purified in Buffer A with a HiLoad 26/60 Superdex 200 prep grade gel filtration column, this extra purification step removes the traces of elution buffer (glutathione or imidazole) as well as further purify the proteins. The purification steps were monitored with SDS PAGE analysis, and the final protein solution yielded a single visible band. The GST-Bxe_A0736 was utilized for the FAC-MS and protein binding microarray experiments and the Bxe_A0736-His 8 was used for the fluorescence anisotropy experiments described below. A representative gel for the expression and purification steps for the GST-Bxe_A0736 fusion protein can be found in the supplemental material, Figure S2.

\subsubsection{FAC-MS Column Preparation}

The GST-Bxe_A0736 protein was diluted to a concentration of $1 \mathrm{mg} / \mathrm{mL}$ and loaded onto a $1 \mathrm{~mL}$ GSTrap FF affinity column. After loading the GST-Bxe_A0736 protein, the FAC-MS columns were washed with 10 column volumes of buffer A. The preparation of the FAC-MS column was concluded by exchanging the binding buffer for a MS friendly buffer such as $20 \mathrm{mM}$ ammonium formate at $\mathrm{pH}$ 7.2.

\subsubsection{Preparation of WT Bxe_A0736}

Wild type (non-tagged) Bxe_A0736 was prepared by cleaving the GST fusion tag from GST-Bxe_A0736 fusion protein by factor Xa from Thermo. The purified fusion protein was loaded onto a $1 \mathrm{~mL}$ GSTrap FF column and equilibrated with factor Xa cleavage buffer composed of $50 \mathrm{mM}$ HEPES, $100 \mathrm{mM} \mathrm{NaCl}, 5 \mathrm{mM} \mathrm{CaCl}, 0.5 \mathrm{mM}$ DTT, pH 7.5. Twenty units of Factor Xa were then dissolved in $1 \mathrm{~mL}$ of the cleavage buffer and loaded onto the column. The column was incubated at room temperature overnight after which the cleaved Bxe_A0736 and Factor Xa were eluted from the column. Factor Xa was removed with Novagen Xarrest agarose beads according to the manufacturer's instructions. The WT Bxe_A0736 was utilized for the DNaseI footprinting experiments described below. A gel showing the purity and approximate size of the WT Bxe_A0736 protein can be found in the supplemental material, Figure S3.

\subsection{Quaternary Structure Determination with Size Exclusion Chromatography}

A Tricorn 5/150 column from GE Healthcare was filled with Superdex 200 prep-grade resin and equilibrated with buffer containing $50 \mathrm{mM}$ Tris, $100 \mathrm{mM} \mathrm{KCl}, \mathrm{pH}$ 7.5. The column was calibrated with a 12-200 kDa molecular weights kit for gel filtration chromatography, purchased from Sigma-Aldrich. To determine the elution volume for each protein in the kit and for WT Bxe_A0736, the protein standards were dissolved in equilibration buffer and WT Bxe_A0736 was concentrated to $1 \mathrm{mg} / \mathrm{mL} .100 \mu \mathrm{L}$ of each protein was injected onto the column. The size of purified WT Bxe_A0736 was then estimated with the use of a calibration curve through comparison of its elution volume to those of the protein size standards.

\subsection{Effector Library Design and Screening with FAC-MS}

Possible effectors were identified based on the frequency of occurrence of those compounds in the KEGG database (http://www.genome.jp/kegg/) as substrates and products of the five genes upstream and downstream of Bxe_A0736 and its twenty closest homologues. Commercially available compounds sharing similar structures to those compounds were included in the library. The compounds tested as effectors included all standard amino acids, 3,4-dihydroxy benzoic acid, 3-hydroxy anthranilic acid, 4-hydroxy benzoic acid, L-kynurenine, 3-hydroxy L-kynurenine as well as the deoxy nucleotide triphosphates, diphosphates and monophosphates.

For effector screening, we utilized our frontal affinity chromatography with ESI-MS detection (FAC-MS) that is designed specifically for screening for TR effector molecules (Marti-Arbona et al., 2012). The affinity column used was GST-Bxe_A0736 fusion protein immobilized on a $1 \mathrm{~mL}$ GSTrap FF column purchased from GE. The 
protein bound column was washed with $20 \mathrm{mM}$ ammonium formate, $\mathrm{pH} 7.4$ for about ten column volumes until the absorbance at $280 \mathrm{~nm}$ stabilized. High-resolution mass detection was performed using a Thermo Exactive ESI-MS equipped with a high-flow steel needle. A typical screening run was performed by infusing mixtures of eight compounds into the column at a rate of $85 \mu \mathrm{L} /$ minute. Each compound was present at $50 \mu \mathrm{M}$ in the ammonium formate buffer. To achieve sufficient positive mode ionization, formic acid-spiked methanol was mixed in equal ratios with the post-column eluent prior to electrospray ionization. The elution times were then noted to rank the affinity of ligands and to identify non-binders that could serve as possible non-ligand void volume markers. The small molecule metabolites have no affinity for the column matrix without immobilized Bxe_A0736.

\subsection{DNA Operator Sequence Identification}

\subsubsection{Protein Binding Microarray Experiments}

A microarray containing all possible 10mer nucleotide combinations was described by Maity and coworkers (Maity et al., 2012). This microarray (available from Agilent) can be used to find a set of sequences for which a DNA binding protein has affinity. These sequences are then analyzed to define a consensus nucleotide sequence bound by the target protein. The GST-Bxe_A0736 fusion protein was bound to the microarray prepared according to the manufacturer's instructions. Protein bound to the microarray was treated with an Alexa488-labeled GST antibody and imaged with the Genepix professional 4200A scanner at resolution of $5 \mu \mathrm{m}$ and an excitation of $488 \mathrm{~nm}$.

\subsubsection{DNaseI Footprinting Experiments}

To prepare DNA material for the DNaseI footprinting experiments, the intergenic region between Bxe_A0736 and Bxe_A0735 plus 60 bp of each flanking gene was PCR amplified from genomic DNA $\overline{-}$ using Alexa488-5'-TGTCGCCTGGCCACACGG-3' primer and the non-tagged reverse primer 5'-TCTTGCTGAAGCACCGTCAAGATACG-3'. This resulted in a PCR product that is a 253 bp piece of Alexa488-labeled, double stranded DNA. The fluorescently labeled DNA was then sequenced using the Thermo Sequenase Cycle Sequencing Kit, according to manufacturer's instructions. The sequencing reactions were analyzed on an ABI 310 genetic analyzer. For the DNase I footprinting reactions, $200 \mathrm{ng}$ of DNA were digested on ice for 5 minutes with 0.004 units of DNase I, with and without various amounts of WT Bxe_A0736 and L-kynurenine. All reactions were performed in Factor Xa cleavage buffer supplemented with $5 \mathrm{mM} \mathrm{MgCl} 2$ and $5 \mathrm{mM}$ DTT. The digested DNA was isolated by phenol/chloroform extraction and ethanol precipitation. For capillary electrophoresis analysis, the DNA samples were de-salted using Bio-Rad Micro Bio-Spin 6 columns, dried and re-suspended in formamide containing $1 \mathrm{mM}$ EDTA and were heated at $95^{\circ} \mathrm{C}$ for $2 \mathrm{~min}$.

\subsection{Fluorescence Anisotropy}

Fluorescence anisotropy was performed with a BioTek Synergy H4 Hybrid Reader, using the standard filter wheel setup for fluorescein. This uses an excitation wavelength of $485 \mathrm{~nm}$ with a 485/20 filter and an emission wavelength of $528 \mathrm{~nm}$ with a $528 / 20$ filter. The reactions took place in a 96 well plate at $30^{\circ} \mathrm{C}$ in $200 \mu \mathrm{L}$ of 25 mM HEPES, $50 \mathrm{mM} \mathrm{KCl}, 25 \mathrm{mM} \mathrm{NaCl}, 2 \mathrm{mM} \mathrm{MgCl}_{2}, 0.1 \mathrm{mg} / \mathrm{mL}$ BSA, pH 7.5, using Bxe_A0736-His $\mathrm{s}_{8}$ and 1 $\mathrm{nM}$ of either of the two $39 \mathrm{bp} 5$ '-fluorescein labeled double-stranded DNA oligos. Each set of oligos contained two sites, representing each half of the protected intergenic region. Listed below in the 5' to 3' direction are the forward sequences of the two oligos. The reverse-complement sequences were label free. All oligos were ordered from Invitrogen.

\section{Oligo1: Fluorescein-5'-TGATAGGTGGCTTGACGCGAAATGTGATTGCGAAAAAAT-3' Oligo2: Fluorescein-5'-ATAATTTGAGATAAATGGGAAAGGGAGACCGAATATGAA-3'}

\subsection{Data Analysis}

Sequence alignments were performed using Clustal W. Consensus sequence analysis was performed using the regulatory sequence analysis tool (Thomas-Chollier et al., 2008). Binding constants were determined by regression analysis using Sigmaplot 12.0. Equation 1 describes the binding of FAC-MS determination of the dissociation constant of L-kynurenine binding to GST-Bxe_A0736 where $V$ is the elution volume of the ligand, $V_{0}$ is the elution volume of a non-ligand void marker and $B_{\mathrm{t}}$ is the amount of immobilized protein with an active binding site (Slon-Usakiewicz, Ng, Dai, Pasternak, \& Redden, 2005).

$$
V-V_{0}=\mathrm{B}_{\mathrm{t}} /\left(\left[\mathrm{A}_{0}\right]+\mathrm{K}_{\mathrm{d}}\right)
$$

Re-arrangement to form a linear expression yields equation 2 where plotting $\left[\mathrm{A}_{0}\right]$ versus $1 /\left(V-V_{0}\right)$ yields a straight line where the slope is $B_{\mathrm{t}}$ and the negative $y$ intercept is $K_{\mathrm{d}}$. 


$$
[\mathrm{A}]_{0}=\mathrm{B}_{\mathrm{t}}\left[1 /\left(V-V_{0}\right)\right]-\mathrm{K}_{\mathrm{d}}
$$

A nonlinear regression analysis of protein concentration versus fluorescence anisotropy was used with equation 3 to determine the kinetic constants for the binding of protein to fluorescein-labeled double stranded DNA. For this equation, $\Delta A$ is the change in anisotropy at a given protein concentration, $\Delta \mathrm{A}_{\mathrm{T}}$ is the total change in anisotropy, $E$ is the protein concentration, $H$ is the Hill coefficient for cooperative binding, and $K_{\mathrm{d}}$ is the dissociation constant for protein-DNA binding (LiCata \& Wowor, 2008).

$$
\Delta \mathrm{A}=\left[\left(\Delta \mathrm{A}_{\mathrm{T}}\left(\mathrm{E}^{H} / \mathrm{K}_{\mathrm{d}}{ }^{H}\right)\right) /\left(1+\left(\mathrm{E}^{H} / \mathrm{K}_{\mathrm{d}}{ }^{H}\right)\right)\right]
$$

\section{Results and Discussion}

\subsection{Sequence Similarity Network Analysis}

The annotation of Bxe_A0736 as asnC, a TR that regulates asparagine synthetase in other bacteria (Kolling \& Lother, 1985; Yokoyama et al., 2005), was not consistent with its conserved close proximity to the genes of the kynurenine pathway (Figure 2). To examine this apparent discrepancy, we first carried out a sequence similarity network analysis (Atkinson et al., 2009) of COG 1522, which contains the diverse members of the AsnC/Lrp TR family in 66 genomes of unicellular organisms (Figure 3). Bxe A0736 and the L-kynurenine responsive TR KynR from $P$. aeruginosa are together in group 24 while AsnC is in group 17. Other TRs of known function, including Lrp, PurR, MdeR and NirH were in separate groups. YbaO and many Grp anotated proteins were in yet another group. Most of the groups contain only a few proteins not associated with any known function. This analysis combined with its genomic proximity with the kynurenine pathway genes encouraged us to experimentally characterize Bxe_A0736 as a potential kynurenine-responsive TR.

\subsection{Native Molecular Weight and Quaternary Structure Examination}

To examine the native oligomeric state of Bxe_A0736, we analyzed the native protein by size exclusion chromatography. According to its elution volume through a calibrated size-exclusion column, the protein eluted as a $183 \pm 2 \mathrm{kDa}$ protein regardless of the presence or absence of L-kynurenine. Given the calculated mass of $19.4 \mathrm{kDa}$ for a single subunit, the estimated molecular weight of $183 \mathrm{kDa}$ corresponds to 9.4 subunits for a globular shaped protein. Thus Bxe-A0736 is an oligomeric protein; an octomeric quaternary structure could be expected (Yokoyama et al., 2005). It also appears that the method may have over estimated the native molecular weight. AsnC/Lrp family of TRs is known to be frequently disc shaped, not the much more familiar spherical shape. Size exclusion chromatography provides good estimates of the native molecular weight for spherical (globular) shaped proteins. However, it over-esimates the molecular weights for disc-shaped proteins because these proteins tumble and therefore move through the column as a function of the diameter of their disc. As a result, they chromatograph like larger spherical proteins. Our data are consistent with Bxe-A0736 being an octameric protein that is disc shaped, like all structurally characterized members of the AsnC/Lrp family of transcriptional regulators.

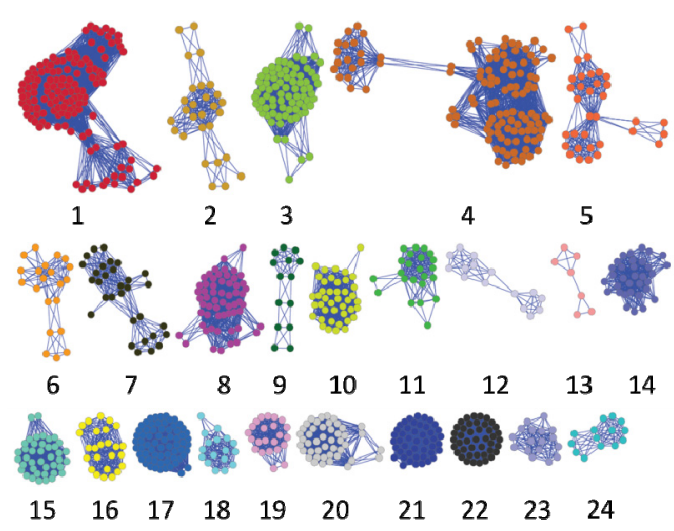

Figure 3. Cytoscape's organic layout sequence similarity network representation for COG1522 which contains AsnC/Lrp family of TRs. The kynurenine-responsive knyR and Bxe_A0736 are together in group 24 while asnC (the current annotation for Bxe_A0736) is in group 17. Each node represents a distinct sequence and each line (edge) is a pairwise connection between two sequences at an $E$ value better than the cutoff 


\subsection{Effector Identification}

We generated a list of possible effectors from the substrates and products listed in the KEGG database for the 5 genes upstream and downstream of the transcriptional regulator Bxe_A0736 and its 40 closest homologues in other bacteria. This list is highly enriched in the tryptophan oxidative metabolic products. The most common metabolite identified is L-formylkynurenine which has 73 occurrences. Anthranilate is the second most common metabolite with 43 occurrences. L-kynurenine, 3-hydroxy-L-kynurenine, formylanthranilate, 3-hydroxyanthranilate and L-alanine all have 41 occurrences. Structural analogues and all amino acids and nucleotides were included in the screen to test for possible effectors.

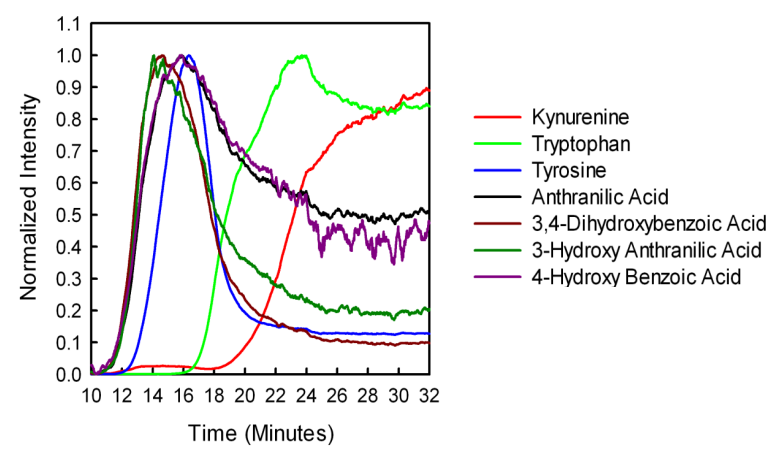

Figure 4. Screening for effector(s) of Bxe_A0736. The elution of the library of potential effectors $(50 \mathrm{uM})$ after flowing past immobilized GST-Bxe-A0736 is shown. The affinity of Bxe_A0736 for these compounds is L-kynurenine $($ red $)>4$-hydroxybenzoic acid $($ dark red $)=$ anthranilic acid (black) $>$ L-tryptophan $($ green $)>$ L-tyrosine (pink) > 3,4-dihydroxybenzoic acid (brown) $=3$-hydroxyanthranilic acid (dark green)

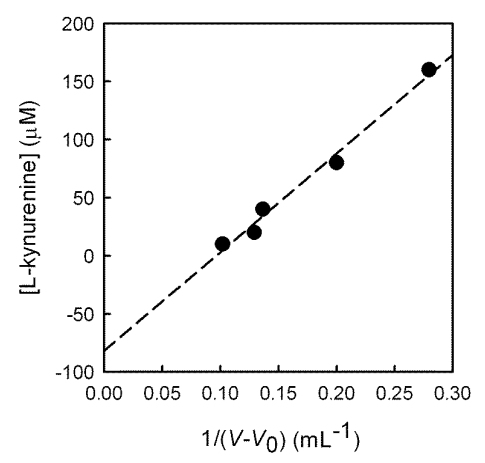

Figure 5. Estimation of the dissociation constant of kynurenine for Bxe_A0736. The elution volumes for kynurenine $(10,20,40,80$ and $160 \mu \mathrm{M})$ when flowed past immobilized GST-Bxe_A0736 were used. The $\mathrm{K}_{\mathrm{d}}$ for L-kynurenine was estimated to be $82 \pm 11 \mu \mathrm{M}$. The column contained $850 \pm 60$ nanomoles of binding sites $\left(\mathrm{B}_{\mathrm{t}}\right)$.

$K_{d}$ and $B_{t}$ were estimated from fits to equation 2

FAC-MS screening identified L-kynurenine, L-phenylalanine, L-tryptophan, L-arginine, and L-lysine as binders of Bxe_A0736. Competition experiments showed that L-kynurenine binds to the immobilized GST-Bxe_A0736 fusion protein with high affinity as shown in Figure 4. The elution volume of L-kynurenine was consistent when present in different mixtures of possible effectors indicating specific binding. L-Phenylalanine, L-tryptophan, L-lysine and L-arginine also showed large elution volumes but were decreased when present in different mixtures, indicating non-specific binding. L-kynurenine was consistently the strongest binder and always had a sigmoidal shaped elution profile. We estimated the dissociation constant for GST-Bxe_A0736 with L-kynurenine to be $82 \pm 11 \mu \mathrm{M}$ (Figure 5). AMP was used as an indicator of the column void volume $\left(V_{0}\right)$ due to its lack of interaction with the column; it was included in all titrations to estimate the breakthrough volume. We found all of the phosphorylated nucleotides were non-binders; they always eluted immediately and with consistent elution volumes. 


\subsection{Identification of Regulatory Sequence}

A protein-binding microarray containing all possible 10mer nucleotides was used to find the set of sequences for which the Bxe_A0736 protein had affinity. By analyzing the 36 sequences for which Bxe_A0736 had affinity, we found a slightly degenerate $\mathrm{A} / \mathrm{T}$ rich $6 \mathrm{bp}$ sequence. The consensus sequence for the forward and reverse sequences of the top 36 binding sequences yields the consensus operator sequence $(\mathrm{C} / \mathrm{G}) \mathrm{ATAT}(\mathrm{A} / \mathrm{G})$ (Figure 6 , panel A).

DNase I footprinting applied to the intergenic region upstream of Bxe_A0735 revealed a rather large protected region composed of $\sim 105$ nucleotides base pairs. This region begins at -35 bp above the ATG start site for kynU. Within this protected region were four areas that showed enhanced protection. Large (up to $200 \mathrm{bps}$ ) regions of DNA involved in binding the TR and containing repeats of a consensus binding site have been observed in other members of this same TR family. These regions for Bxe-A0735 are illustrated in Figure 7.

Analysis of the four protected regions in the intergenic sequence did not yield an obvious consensus sequence, so we evaluated a sequence alignment of homologous intergenic regions from multiple Burkholderia species. The conserved regions from the eight closest homologues shown in Figure 7 correspond to the areas of enhanced protection. Utilizing these conserved and protected regions, we found the degenerate palindromic consensus sequence shown in the panel B of Figure 6. The degenerate sequence is: DBHDDNSNHHDVH where $\mathrm{D}=\mathrm{G}, \mathrm{A}$ or $\mathrm{T} ; \mathrm{B}=\mathrm{G}, \mathrm{C}$ or $\mathrm{T} ; \mathrm{H}=\mathrm{A}, \mathrm{C}$ or $\mathrm{T} ; \mathrm{N}=$ any nucleotide; $\mathrm{S}=\mathrm{C}$ or $\mathrm{G}$; and $\mathrm{V}=\mathrm{G}, \mathrm{A}$ or $\mathrm{C}$. Based on the most common base found at each position, the optimal sequence is: ATATTCCGAATAT. This sequence is similar to the smaller half-site consensus sequence found using the protein-binding microarray. The fact that the sequences of these protein-binding regions are not highly conserved may be an indication that the TR recognizes common features of the DNA structure that may form from various DNA sequences.
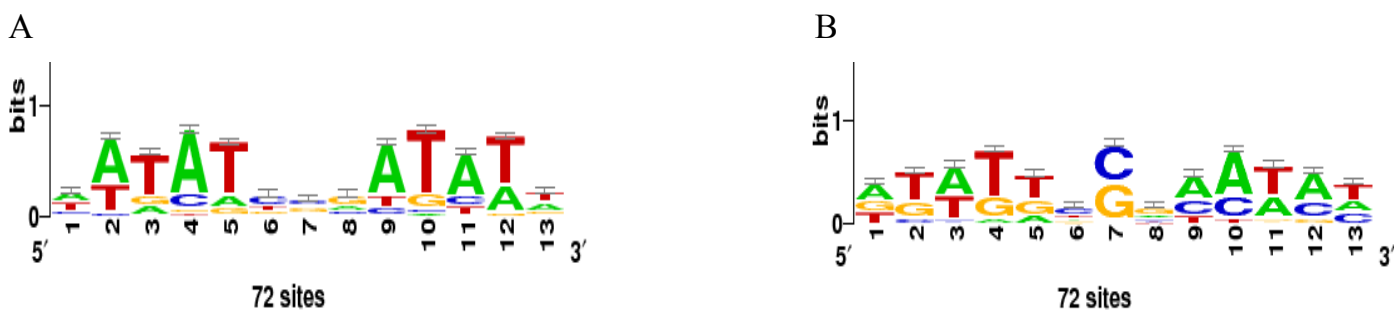

Figure 6. Consensus sequences for the regulatory sites derived for Bxe_A0736. Panel A shows the consensus sequence as determined from the protein binding microarray.et al.,Panel B shows the consensus sequence of the four binding sites protected during the phylogenetic footprinting using the intergenic sequence upstream from Bxe_A0736 and the eight homologues from different Burkholderia species

\subsection{Fluorescence Anisotropy}

Two oligos were constructed and used to test if the presence of kynurenine affected the binding by Bxe_0736 to portions of the much larger DNA region identified to interact with this TR. Because fluorescence anisotropy cannot be used with large DNA sequences, we divided the DNA interaction region into two sequences (oligos1 and 2); each contained two of the proposed binding sites. For each oligo with Bxe_A0736-His 8 , the presence of L-kynurenene increased the affinity (ie decreased the dissociation constant) for the oligo. The titration of $1 \mathrm{nM}$ fluorescein labeled oligo 1 with Bxe_A0736- $\mathrm{His}_{8}$, yielded an apparent dissociation constant of $145 \mathrm{nM}$ for Bxe_A0736-His 8 . With $1 \mathrm{mM} \mathrm{L-kynurenine} \mathrm{present,} \mathrm{the} \mathrm{dissociation} \mathrm{constant} \mathrm{was} \mathrm{lowered} \mathrm{to} 97 \mathrm{nM}$. The Hill coefficient was also lowered from 5.2 to 4.2 with L-kynurenine. Examination of Bxe_A0736-His 8 with $1 \mathrm{nM}$ oligo2 yielded a dissociation constant of $154 \mathrm{nM}$ and a Hill coefficient of $5.3 \mathrm{In}$ the presence of $1 \mathrm{mM}$ L-kynurenine, the dissociation constant was reduced to $128 \mathrm{nM}$ and the Hill coefficient was lowered to 4.8. Even when higher concentrations of $\mathrm{NaCl}, \mathrm{KCl}$ or $\mathrm{MgCl}_{2}$ were used in the assay, the presence of L-kynurenine lowered the dissociation constant and Hill coefficient. The values obtained with these oligos cannot be expected to quantitatively reflect the affinity of Bxe_A0736 for the entire interaction region that contained four, not two binding sites as did the oligos. These values can be expected to be under estimations. In addition, the conditions of the experiment may not accurately reproduce the intracellular conditions. The data do support L-kynurenene increasing the affinity of Bxe_A0736 for the identified binding sites. 


\section{GENE NAME}

BPHY 0509

Bxe_A0736

BPHYT_3226

BC1002_2483

BH160DRAFT_4294

BOKLC_010100004123

BUBOB_010100009881

BAMMEX5DRAFT_5259

BCAL2789

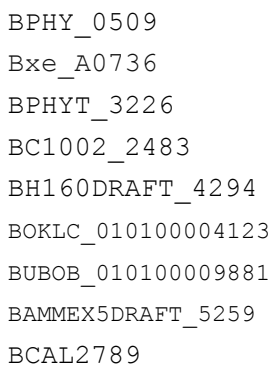

\section{$\chi_{\text {Kynurenine Formamidase }(k y n B)}$}

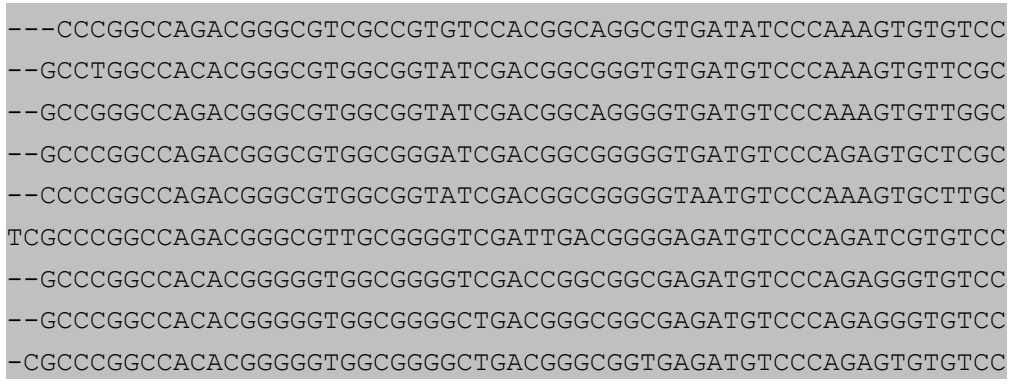

ATGT-GTCCCCGGTGCATCTGGCAGCTAT-GGAACGATGAtAGGCCGCATCAGACGAAAT
ATAGTCGCATCGGCAGTTTGTG--TCTAT-CCGGGAATGATAGGTGGCTTGACGCGAAAT
ATAGTCGCATCGGCGGTTTGTG--TCTAT-CCGGGAATGATAGGTGGCTTGCCGCGAAT
ATGACGGTTTCGACGGAAGGTG--ACTAT-CTCTAAATGATAGAGGTCAAGCCGTGAAAT
ATGACGGTTTCGGCGAAAGGTG--ACTCT-CTTTGAATGATAGAGGTCAAGCCGCGAAAT
ATGC-GATATCGAAGAACGTTG----GATGCTTCGGATGATAGGCAAACCTGCGAAGCAG
ATGG-CATGGCGAAG--CAGCGAAATGATGCTTCGCATGATAGGCGGACGCTCGCAAAAA
ATGG-CATGACGAAT--CGGTGAATCGATCCTTCGAATGATAGGTGGACACTCGCAGAAA
ATGG-CATGACGAAG---CGTGAGTCGATCCTTCGAATGATAGGTGGACGCTCGCAGAAA

GTGCTTGCTAAAAAAACTGTTTATTTCGCCCCCTTTGGAACATAATTTGAGGCAAACGGG GTGATTGCGAAAAAATCTCCTTCCAGGCCGTGTCTTGGAACATAATTTGAGATAAATGGG GTGATTGCGAAAAAATCTCCTTCTGCGCCGTGTCTTGGAACATAATTTGATTCAAACGGG GTGATTGCGAAAAAATCTCCTAACCAGCCGTGTCTTGGAACATAATTTGAGCCAAACCGG GTGATTGCGAAAAAATCTCCTTCCTAGCCGTGTCTTGGAACATAATTTGAGTCAAACAGG GTGCTTGCGAAATAAGCATGGACAATCCACCAATATGGCAGATAATTCGAAATAGACCTA GTGCTTGCGAAATAACCATGGACAAACCGCCAGATTGGCAGATAATTCGAAGCAGAGAGG GTGCTTGCGAAATAAACATGGACAATCCGCCGGATTGGCAGATAATTCGAATGAGAGGGA GTGCTTGCGAAATAAACATGGACAATCCGCCGGATTGGCAGATAATTCGACTCAGAGGGA

$$
\begin{aligned}
& \text { AAAGGGAGACCGAAAATGAACGCGATCTCACTAGACGCCACCGATTGCCGTATCTTGACG } \\
& \text { AAAGGGAGACCGAATATGAACGCGATCTCGCTCGACGCCACCGATTGCCGTATCTTGACG } \\
& \text { AAAGGGAGACCGAATATGAACGCGATCTCGCTCGACGCCACCGATTGCCGTATCTTGACG } \\
& \text { AAATGGAGACCGAATATGAACGCGATCTCGCTCGACGCCACCGATTGCCGTATCTTGACG } \\
& \text { AAAGGGAGACCGAATATGAACGCGATCTCGCTCGACGCCACCGATTGCCGTATCTTGACG } \\
& \text { AAACGGAGACCAAAAATGCATGCGATCACGCTTGACGCAACCGATTGCCGTATTCTCGCG } \\
& \text { AAACGGAGACCAAAAATGCACGCGATCACGCTCGACGCCACCGACTGCCGGATTCTCGCG } \\
& \text { AAACGGGGACCAAAAATGCACGCGATCACGCTCGACGCCACCGACTGCAGGATTCTGGCG } \\
& \text { AAACGGGGACCAAAAATGCACGCGATCACGCTCGATGCCACCGACTGCCGGATTCTGGCG }
\end{aligned}
$$

Figure 7. Multiple sequence alignment of the intergenic regions between kynurenine formamidases and the L-kynurenine responsive AsnC/Lrp like transcriptional regulators. The protein coding regions are highlighted in gray. The four degenerate palindromic sequences identified by DNasel footprinting are underlined

\section{Conclusions}

Our results support that Bxe_A0736 is a TR of the kynurenine pathway of L-tryptophan degradation in $B$. xenovorans and that it should be designated as KynR. Bxe_A0736 (KynR) interacts with a rather long region of DNA that contains four small repeated pallindromic sequences for which a consensus sequence could be defined. The presence of kynurenine was accompanied by a moderate increase in affinity of Bxe_A0736 for a portion of the much longer region with which it interacts. One of the small pallindromic regulatory sites for Bxe_A0736 (KynR) lies over the ATG start codon for kynR, suggesting that Bxe_A0736 (KynR) binding may block its own transcription. 


\section{Acknowledgements}

We thank Dr. Virginia A. Unkefer for editorial improvements to this manuscript. This work was conducted in part under the auspices of the US Department of Energy and supported by the LDRD program at the Los Alamos National Laboratory (Grant No. 20090107DR).

\section{References}

Atkinson, H. J., Morris, J. H., Ferrin, T. E., \& Babbitt, P. C. (2009). Using sequence similarity networks for visualization of relationships across diverse protein superfamilies, PLoS One, 4(2), e4345. http://dx.doi.org/10.1371/journal.pone.0004345

Belladonna, M. L., Orabona, C., Grohmann, U., \& Puccetti, P. (2009). TGF-beta and kynurenines as the key to infectious tolerance, Trends Mol Med, 15(2), 41-49. http://dx.doi.org/S1471-4914(09)00013-6

Brinkman, A. B., Ettema, T. J., de Vos, W. M., \& van der Oost, J. (2003). The Lrp family of transcriptional regulators. Mol Microbiol, 48(2), 287-294. http://dx.doi.org/3442

Calvo, J. M., \& Matthews, R. G. (1994). The leucine-responsive regulatory protein, a global regulator of metabolism in Escherichia coli, Microbiol Rev, 58(3), 466-490.

Cline, M. S., Smoot, M., Cerami, E., Kuchinsky, A., Landys, N., Workman, C., ... Bader, G. D. (2007). Integration of biological networks and gene expression data using Cytoscape. Nat Protoc, 2(10), 2366-2382. http://dx.doi.org/nprot.2007.324

De Wind, N., de Jong, M., Meijer, M., \& Stuitje, A. R. (1985). Site-directed mutagenesis of the Escherichia coli chromosome near oriC: identification and characterization of asnC, a regulatory element in E. coli asparagine metabolism. Nucleic Acids Res, 13(24), 8797-8811.

Enoru-Eta, J., Gigot, D., Thia-Toong, T. L., Glansdorff, N., \& Charlier, D. (2000). Purification and characterization of Sa-lrp, a DNA-binding protein from the extreme thermoacidophilic archaeon Sulfolobus acidocaldarius homologous to the bacterial global transcriptional regulator Lrp. J Bacteriol, 182(13), 3661-3672.

Farrow, J. M., \& Pesci, E. C. (2007). Two distinct pathways supply anthranilate as a precursor of the Pseudomonas quinolone signal. J Bacteriol, 189(9), 3425-3433. http://dx.doi.org/JB.00209-07

Inoue, H., Inagaki, K., Eriguchi, S. I., Tamura, T., Esaki, N., Soda, K., \& Tanaka, H. (1997). Molecular characterization of the mde operon involved in L-methionine catabolism of Pseudomonas putida. J Bacteriol, 179(12), 3956-3962.

Keuntje, B., Masepohl, B., \& Klipp, W. (1995). Expression of the putA gene encoding proline dehydrogenase from Rhodobacter capsulatus is independent of $\mathrm{NtrC}$ regulation but requires an Lrp-like activator protein. $J$ Bacteriol, 177(22), 6432-6439.

Knoten, C. A., Hudson, L. L., Coleman, J. P., Farrow, J. M., \& Pesci, E. C. (2011). KynR, a Lrp/AsnC-Type Transcriptional Regulator, Directly Controls the Kynurenine Pathway in Pseudomonas aeruginosa. $J$ Bacteriol, 193(23), 6567-6575. http://dx.doi.org/JB.05803-11

Koike, H., Ishijima, S. A., Clowney, L., \& Suzuki, M. (2004). The archaeal feast/famine regulatory protein: potential roles of its assembly forms for regulating transcription. Proc Natl Acad Sci U S A, 101(9), 2840-2845. http://dx.doi.org/10.1073/pnas.0400109101

Kolling, R., \& Lother, H. (1985). AsnC: an autogenously regulated activator of asparagine synthetase A transcription in Escherichia coli. J Bacteriol, 164(1), 310-315.

Kolling, R., \& Lother, H. (1985). AsnC: an autogenously regulated activator of asparagine synthetase A transcription in Escherichia coli. J Bacteriol, 164(1), 310-315.

Landgraf, J. R., Wu, J., \& Calvo, J. M. (1996). Effects of nutrition and growth rate on Lrp levels in Escherichia coli. J Bacteriol, 178(23), 6930-6936.

LiCata, V. J., \& Wowor, A. J. (2008). Applications of fluorescence anisotropy to the study of protein-DNA interactions. Methods Cell Biol, 84, 243-262. http://dx.doi.org/S0091-679X(07)84009-X

Madhusudhan, K. T., Lorenz, D., \& Sokatch, J. R. (1993). The bkdR gene of Pseudomonas putida is required for expression of the bkd operon and encodes a protein related to Lrp of Escherichia coli. J Bacteriol, 175(13), 3934-3940. 
Maity, T. S., Close, D. W., Valdez, Y. E., Nowak-Lovato, K., Marti-Arbona, R., Nguyen, T. T., ... Dunbar, J. (2012). Discovery of DNA operators for TetR and MarR family transcription factors from Burkholderia xenovorans. Microbiology, 158(Pt 2), 571-582. http://dx.doi.org/10.1099/mic.0.055129-0

Marti-Arbona, R., Teshima, M., Anderson, P. S., Nowak-Lovato, K. L., Hong-Geller, E., Unkefer, C. J., \& Unkefer, P. J. (2012). Identification of new ligands for the methionine biosynthesis transcriptional regulator (MetJ) by FAC-MS. J Mol Microbiol Biotechnol, 22(4), 205-214. http://dx.doi.org/10.1159/000339717

Matthijs, S., Baysse, C., Koedam, N., Tehrani, K. A., Verheyden, L., Budzikiewicz, H., ... Cornelis, P. (2004). The Pseudomonas siderophore quinolobactin is synthesized from xanthurenic acid, an intermediate of the kynurenine pathway. Mol Microbiol, 52(2), 371-384. http://dx.doi.org/10.1111/j.1365-2958.2004.03999.x

Palleroni, N. J., \& Stanier, R. Y. (1964). Regulatory mechanisms governing synthesis of the enzymes for tryptophan oxidation by Pseudomonas fuorescens. J Gen Microbiol, 35, 319-334.

Peekhaus, N., Tolner, B., Poolman, B., \& Kramer, R. (1995). The glutamate uptake regulatory protein (Grp) of Zymomonas mobilis and its relation to the global regulator Lrp of Escherichia coli. J Bacteriol, 177(17), 5140-5147.

Ren, J., Sainsbury, S., Combs, S. E., Capper, R. G., Jordan, P. W., Berrow, N. S., ... Owens, R. J. (2007). The structure and transcriptional analysis of a global regulator from Neisseria meningitidis. J Biol Chem, 282(19), 14655-14664. http://dx.doi.org/M701082200

Slon-Usakiewicz, J. J., Ng, W., Dai, J. R., Pasternak, A., \& Redden, P. R. (2005). Frontal affinity chromatography with MS detection (FAC-MS) in drug discovery. Drug Discov Today, 10(6), 409-416. http://dx.doi.org/S1359644604033604

Tatusov, R. L., Fedorova, N. D., Jackson, J. D., Jacobs, A. R., Kiryutin, B., Koonin, E. V., .. Natale, D. A. (2003). The COG database: an updated version includes eukaryotes. BMC Bioinformatics, 4(41). http://dx.doi.org/10.1186/1471-2105-4-41

Thaw, P., Sedelnikova, S. E., Muranova, T., Wiese, S., Ayora, S., Alonso, J. C., ... Rafferty, J. B. (2006). Structural insight into gene transcriptional regulation and effector binding by the Lrp/AsnC family. Nucleic Acids Res, 34(5), 1439-1449. http://dx.doi.org/34/5/1439

Thomas-Chollier, M., Sand, O., Turatsinze, J. V., Janky, R., Defrance, M., Vervisch, E., ... van Helden, J. (2008). RSAT: regulatory sequence analysis tools. Nucleic Acids Res, 36(Web Server issue), W119-127. http://dx.doi.org/gkn304

Wang, Q., \& Calvo, J. M. (1993). Lrp, a global regulatory protein of Escherichia coli, binds co-operatively to multiple sites and activates transcription of ilvIH. $J$ Mol Biol, 229(2), 306-318. http://dx.doi.org/S0022-2836(83)71036-3

Willins, D. A., Ryan, C. W., Platko, J. V., \& Calvo, J. M. (1991). Characterization of Lrp, an Escherichia coli regulatory protein that mediates a global response to leucine. J Biol Chem, 266(17), 10768-10774.

Yokoyama, K., Ebihara, S., Kikuchi, T., \& Suzuki, M. (2005). Binding of the feast/famine regulatory protein (FFRP) FL11 (pot0434017) to DNA in the "promoter to coding" region of gene fl11. Proceedings of the Japan Academy Series B-Physical and Biological Sciences, 81(2), 64-75. 


\section{S. Supplementary Information}

\section{S.1 Bxe_A0736: GST Fusion Purification}

The $E$. coli optimized nucleotide sequence for Bxe_A0736 is shown in Figure S1.

$$
\begin{aligned}
& \text { ATGAATGCGATCTCCCTGGACGCGACGGACTGCCGTATCCTGACGGTTCTGCAGCAAGAAGGCCGTATCTCGAA } \\
& \text { TCTGGACCTGGCGGAACGTATTAGCCTGTCTCCGAGTGCATGCCTGCGTCGCCTGCGTCTGCTGGAAGAACAGG } \\
& \text { GCGTCATCGAACATTATCGTGCATGTCTGAACCGCGAAGTGCTGGGTTTTGAACTGGAAGCTTTCGTTCAGGTC } \\
& \text { TCGATGCGTAACGATCAAGAAAATTGGCATGAACGTTTTGCAGATGCAGTGCGTGACTGGCCGGAAGTGGTTGG } \\
& \text { TGCGTTCGTCGTGACCGGTGAAACGCACTACCTGCTGCGCGTTCTGGCCCATAACCTGAAACACTATTCTGACT } \\
& \text { TTGTCCTGCAGCGTCTGTACAAAGCCCCGGGCGTGATGGATATTCGCTCCAATATCGTTCTGCAAACCCTGAAA } \\
& \text { GAAGACTCAGGTGTTCCGGTTTCACTGGTCAAAAAAGCATCGGGTCACGGCGCTGCCCATAATGATCGCTAA }
\end{aligned}
$$

Figure S1. Coding DNA sequence for Bxe_A0736 optimized for E. coli expression

\section{S.2 Expression and Purification of the GST-Bxe_A0736}

As described in the manuscript, the purification of the GST-Bxe_A0736 fusion protein involved two steps. The first step was affinity chromatography using a GSTrap FF HiTrap affinity column. Protein that bound to the affinity resin was eluted with a glutathione gradient and fractions containing protein were pooled, concentrated and chromatographed using a HiLoad 26/60 Superdex 200 prep grade gel filtration column. This extra purification step removes the traces of the glutathione elution buffer as well as further purifies the protein. The purification was monitored using SDS PAGE analysis (Figure S2). The final protein solution yielded predominately a single visible band, with electrophoretic mobility consistent calculated mass of $50.7 \mathrm{kDa}$ for the GST-Bxe_A0736 fusion protein.

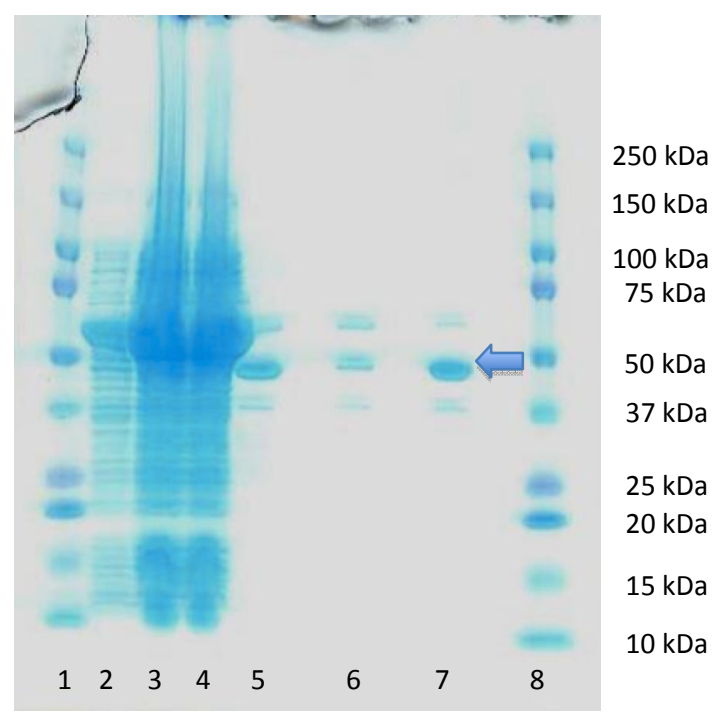

Figure S2. SDS PAGE analysis of the purification of the GST-Bxe_A0736 fusion protein. Lanes 1 and 8 show the positions of molecular mass standards labeled on the right. Lane 2 shows the protein profile of E. coli induced to express the GST-Bxe_A0736. Lane 3 shows the protein profile of the same $E$. coli cells lysed for protein purification. Lane 4 shows the protein profile of the solution that flows through the GST affinity column. Lane 5 shows the protein profile of the pooled protein-containing fractions eluted from the affinity resin with reduced glutathione. Two protein-containing fractions that eluted from the gel filtration column were pooled independently and their protein profiles are represented in lanes 6 and 7. Lane 7 represents the purified solution of the GST-Bxe_A0736 fusion protein that was used for our experiments. The blue arrow points to the band corresponding to the GST-Bxe_A0736 fusion protein 


\section{S3: Preparation of WT Bxe_A0736}

As described in the manuscript, the GST-Bxe_A0736 was bound to a GSTrap FF HiTrap affinity column and incubated at room temperature with twenty units of Factor Xa. After overnight incubation the cleaved Bxe_A0736 and Factor Xa were eluted from the column. Factor Xa was removed with Novagen Xarrest agarose beads according to the manufacturer's instructions. The cleavage and purification were monitored with SDS PAGE analysis shown in Figure 3S. The cleaved protein solution yielded a single visible band whose electrophoretic mobility was consistent with the calculated mass of $19.4 \mathrm{kDa}$ for the Bxe_A0736.

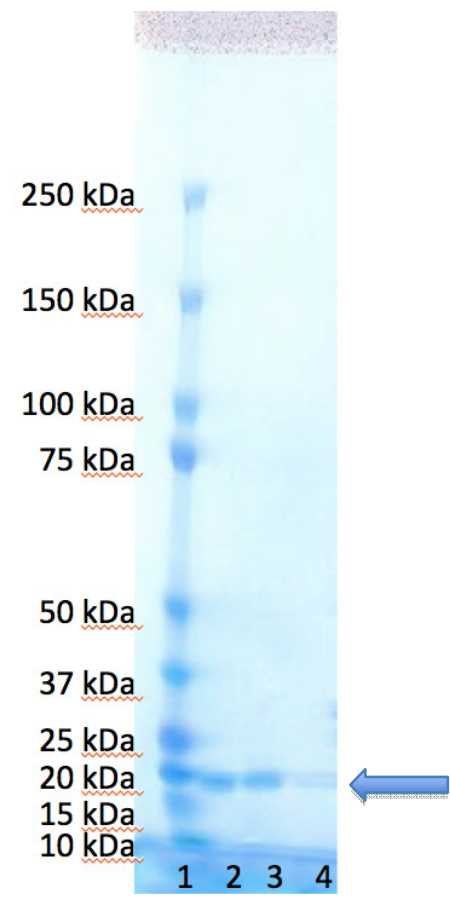

Figure S3. SDS PAGE analysis of the cleavage of Bxe_A7036 from the Bxe_A7036:GST fusion protein. Lanes 1 shows the electrophoretic mobility of molecular mass standards labeled on the left. Lane 2, 3 and 4 show the protein profile of the cleaved product after removal of Factor Xa. The blue arrow points to the band corresponding to the GST-Bxe_A0736 fusion protein

\section{Copyrights}

Copyright for this article is retained by the author(s), with first publication rights granted to the journal.

This is an open-access article distributed under the terms and conditions of the Creative Commons Attribution license (http://creativecommons.org/licenses/by/3.0/). 